\title{
The ping-pong game, geometric entropy and expansiveness for group actions on Peano continua having free dendrites
}

by

\author{
Enhui Shi and Suhua Wang (Suzhou)
}

\begin{abstract}
It is shown that each expansive group action on a Peano continuum having a free dendrite must have a ping-pong game, and has positive geometric entropy when the acting group is finitely generated. As a corollary, it is shown that each Peano continuum having a free dendrite admits no expansive nilpotent group actions.
\end{abstract}

1. Introduction and preliminaries. Let $X$ be a topological space and let $G$ be a topological group. Recall that a continuous map $\phi: G \times X \rightarrow X$ is called an action of $G$ on $X$ if the following two conditions are satisfied:

(1) $\phi(e, x)=x$ for all $x \in X$, where $e$ is the identity of $G$.

(2) $\phi\left(g_{1} g_{2}, x\right)=\phi\left(g_{1}, \phi\left(g_{2}, x\right)\right)$ for all $g_{1}, g_{2} \in G$, and all $x \in X$.

Such an action is denoted by the triple $(X, G, \phi)$. For convenience, we always use $g x$ or $g(x)$ to denote $\phi(g, x)$. Obviously, for each $g \in G$, the map $g$ : $X \rightarrow X, x \mapsto g x$, is a homeomorphism on $X$.

Throughout the paper, we assume that $G$ has the discrete topology.

The orbit of $x \in X$ is the set $G x=\{g x: g \in G\}$. For a subset $A \subseteq X$, set $G A=\bigcup_{x \in A} G x$. A nonempty subset $A \subseteq X$ is said to be $G$-invariant if $G A=A$. If $A$ is $G$-invariant, then $\left.G\right|_{A}$ denotes the action of $G$ restricted to $A$.

Now, let $G$ be a discrete group acting on a topological space $X$. We are interested in the following question: what are the relations between

$\diamond$ the topology of $X$,

$\diamond$ the algebraic structure of $G$, and

$\diamond$ the dynamics of the action?

Before the statement of the main results, let us recall some definitions.

2000 Mathematics Subject Classification: Primary 54H20; Secondary 37B05.

Key words and phrases: Peano continuum, expansiveness, group action, geometric entropy, ping-pong game, free dendrite. 
Continuum. A continuum is a connected, compact metric space. A continuum $X$ is said to be a Peano continuum if it is locally connected. A dendrite is a Peano continuum having no simple closed curve. A dendrite $D$ in a metric space $X$ is free if there exists a connected open subset $U$ of $X$ such that $\bar{U}=D$. One may consult [13] for the details of these notions.

Group. The following definitions are taken from [5]. Suppose that $G$ is a group and $a, b \in G$. Recall that the commutator $[a, b]$ is defined by $[a, b]=a^{-1} b^{-1} a b$. For any two subsets $A$ and $B$ of $G$, define $[A, B]$ to be the subgroup generated by the set $\{[a, b]: a \in A, b \in B\}$. Let $G_{0}=G$ and $G_{i+1}=\left[G_{i}, G\right]$ for $i=0,1,2, \ldots$ This yields a sequence of normal subgroups of $G: G_{0}=G \triangleright G_{1} \triangleright G_{2} \triangleright \cdots$. If $G_{n}=e$ for some $n$, then $G$ is called nilpotent. It is well known that each subgroup of a nilpotent group is nilpotent and nilpotent groups have no free subsemigroups.

Dynamics. Let $X$ be a compact metric space with metric $d$ and let $\phi: G \times X \rightarrow X$ be a group action. If there is a constant $c>0$ such that for any distinct points $x, y \in X$, there is some $g \in G$ such that $d(g(x), g(y))>c$, then $\phi$ is said to be expansive, and $c$ is said to be an expansive constant for $\phi$. A homeomorphism $f$ of $X$ is called expansive if the cyclic group generated by $f$ is expansive, that is, there exists $c>0$ such that for any $x, y \in X$ with $x \neq y$, there is an $n \in \mathbb{Z}$ with $d\left(f^{n}(x), f^{n}(y)\right)>c$.

Consider a finitely generated group $G$. Choose a symmetric generating set $S=\left\{\sigma_{0}, \sigma_{1}, \ldots, \sigma_{k}\right\}$ for $G$, where $\sigma_{0}$ is the identity. The symmetry means here that for each $1 \leq i \leq k, \sigma_{i}^{-1}=\sigma_{l}$ for some $1 \leq l \leq k$. An element $g \in G$ has word length $\|g\| \leq N$ if there exist indices $i_{1}, \ldots, i_{N}$ such that $g=\sigma_{i_{1}} \cdots \sigma_{i_{N}}$. The definition of geometric entropy of a group action $\phi: G \times X \rightarrow X$ is given by Ghys, Langevin and Walczak [2]. Given $\varepsilon>0$ and an integer $N>0$, we say that $x, y \in X$ are $(N, \varepsilon)$-separated if there exists $g \in G$ with $\|g\| \leq N$ and $d(g x, g y)>\varepsilon$. A finite subset $\left\{x_{1}, \ldots, x_{\nu}\right\} \subset X$ is said to be $(N, \varepsilon)$-separated if for any $k \neq l$, the points $x_{k}, x_{l}$ are $(N, \varepsilon)$-separated. Let $S(\phi, \varepsilon, N)$ denote the maximum cardinality of an $(N, \varepsilon)$-separated subset of $X$. Now define

$$
h(\phi, \varepsilon)=\limsup _{N \rightarrow \infty} \frac{\log S(\phi, \varepsilon, N)}{N} \geq 0 .
$$

The geometric entropy of $\phi$ is the limit $h(\phi)=\lim _{\varepsilon \rightarrow 0} h(\phi, \varepsilon) \geq 0$.

We next introduce a dynamical concept which is called a ping-pong game. This concept goes back to the work of Blaschke, Klein, Schottky and Poincaré (see [3]). Let $G$ be a group acting on the compact metric space $X$ and let $J, J_{1}, J_{2}$ be closed subsets of $X$ such that $J_{1}, J_{2} \subset J$ and $J_{1} \cap J_{2}=\emptyset$. If $g_{1}, g_{2} \in G$ satisfy $g_{1} J \subset J_{1}, g_{2} J \subset J_{2}$, then the pair of maps $\left\{g_{1}: J \rightarrow J_{1}\right.$, $\left.g_{2}: J \rightarrow J_{2}\right\}$ is called a ping-pong game for the action. 
Expansiveness, ping-pong game and positive geometric entropy can each be viewed as a complicated dynamical property.

In this paper, we show the following:

THEOREM 1.1. Each expansive group action on a Peano continuum containing a free dendrite must have a ping-pong game, and has positive geometric entropy when the acting group is finitely generated.

Corollary 1.2. No Peano continuum having a free dendrite admits an expansive nilpotent group action.

REMARK 1.3. The above results have been proved by S. Hurder when the space is the circle $\mathbb{S}^{1}$ (see [4]), which answers the question whether $\mathbb{S}^{1}$ admits an expansive nilpotent group action proposed by T. Ward in 1999 (see $[10])$.

REMARK 1.4. Whether a continuum admits an expansive homeomorphism is an interesting problem in topological dynamics and continuum theory. One may consult $[1,6-9,12,14,16]$ for developments in this area. Recently some authors are interested in the existence of expansive group actions on continua, and some results have been obtained (see $[11,15]$ ).

2. Peano continua containing free dendrites. In this section, we will give some topological properties of Peano continua which will be used in the proof of the main theorem.

First let us recall some notions. Let $Y$ be a subset of $X$. The symbols $\bar{Y}$, Int $(Y)$, and $\partial_{X}(Y)$ stand for the closure, interior, and boundary of $Y$ in $X$ respectively. A cut point of a connected space $X$ is a point $p \in X$ such that $X-\{p\}$ is not connected. The set of cut points of $X$ will be denoted by $\operatorname{Cut}(X)$. An endpoint of a space $X$ is a point of $X$ that has arbitrarily small neighborhoods in $X$ with one-point boundaries. The symbol $\operatorname{End}(X)$ denotes the set of all endpoints of $X$.

Let $D$ be a dendrite, and $A$ be an arc in $D$. Write $\AA=A-\operatorname{End}(A)$. Then $D(A)=A \cup \bigcup\{Y: Y$ is a component of $D-A$, and $\bar{Y} \cap \AA \neq \emptyset\}$ is called the subdendrite of $D$ strung by $A$.

Lemmas 2.1, 2.3 and 2.4 have been proved in [11]. For completeness and for the convenience of the reader, we include the proofs of the last two lemmas; the first one is clear.

Lemma 2.1 ([11]). Let $D$ be a dendrite, $A$ an arc in $D$, and $D_{1}=D(A)$ the subdendrite of $D$ strung by $A$.

(1) If $A^{\prime}$ is a subarc of $A$, then $D_{1}\left(A^{\prime}\right)=D\left(A^{\prime}\right)$.

(2) If $A^{\prime}$ and $A^{\prime \prime}$ are subarcs of $A$ and $A^{\prime} \cap A^{\prime \prime}=\emptyset$, then $D\left(A^{\prime}\right) \cap$ $D\left(A^{\prime \prime}\right)=\emptyset$. 
Definition 2.2 ([11]). A free dendrite $D$ in a metric space $X$ is then said to be strongly free if there is an arc $A \subset D$ such that $\partial_{X}(D)=\operatorname{End}(A) \subset$ $\operatorname{End}(D)$. The $\operatorname{arc} A$ is then said to be the trunk of $D$.

For any $y \in Y \subset X$ and any $\varepsilon>0$, write $B(y, \varepsilon)=\{x \in X: d(x, y)<\varepsilon\}$ and $B(Y, \varepsilon)=\{x \in X: d(x, Y)<\varepsilon\}$.

Lemma 2.3 ([11]). A metric space $X$ contains a strongly free dendrite if and only if it contains a free dendrite.

Proof. We need only prove the sufficiency. Let $U$ be a connected open set in $X$ such that $\bar{U}$ is a dendrite. Take an $\operatorname{arc} D$ in $X$ and an $\varepsilon>0$ such that $\overline{B(D, \varepsilon)} \subset U$. Since $\bar{U}-D$ has only finitely many components of diameter $>\varepsilon$, there exists an arc $A \subset D-\operatorname{End}(D)$ such that the closure of each component of $\bar{U}-D$ which has diameter $>\varepsilon$ is disjoint from $A-\operatorname{End}(A)$. Let $D_{1}=\bar{U}(A)$ be the subdendrite of $\bar{U}$ strung by $A$. Then $D_{1} \subset \overline{B(A, \varepsilon)} \subset$ $\overline{B(D, \varepsilon)} \subset U$, and $\partial_{\bar{U}}\left(D_{1}\right)=\operatorname{End}(A) \subset \operatorname{End}\left(D_{1}\right)$. Hence $D_{1}-\operatorname{End}(A)$ is open in $\bar{U}$ (in the relative topology of $\bar{U}$ ), and thus $D_{1}-\operatorname{End}(A)$ is open in $U$ (in the relative topology of $U)$. Since $U$ is an open subset of $X, D_{1}-\operatorname{End}(A)$ is open in $X$. Thus $\partial_{X}\left(D_{1}\right) \subset \operatorname{End}(A)=\partial_{\bar{U}}\left(D_{1}\right) \subset \partial_{X}\left(D_{1}\right)$, and so $D_{1}$ is a strongly free dendrite in $X$.

Lemma 2.4 ([11]). Suppose $D$ is a strongly free dendrite in the metric space $X$ with $A$ being its trunk, and $A^{\prime}$ is a subarc of $A$. Then $D\left(A^{\prime}\right)$ is a strongly free dendrite in $X$ with $A^{\prime}$ being its trunk.

Proof. Obviously $D\left(A^{\prime}\right)-\operatorname{End}\left(A^{\prime}\right)$ is an open subset of $D$. So it is an open subset of the space $D-\partial_{X}(D)$, and of the space $X$. This implies $\partial_{X}\left(D\left(A^{\prime}\right)\right) \subset \operatorname{End}\left(A^{\prime}\right)$. On the other hand, whether $\operatorname{End}\left(A^{\prime}\right) \cap \operatorname{End}(A)=\emptyset$ or not, we always have $\operatorname{End}\left(A^{\prime}\right) \subset \partial_{X}\left(D\left(A^{\prime}\right)\right)$, which implies $\partial_{X}\left(D\left(A^{\prime}\right)\right)=$ $\operatorname{End}\left(A^{\prime}\right) \subset \operatorname{End}\left(D\left(A^{\prime}\right)\right)$. Thus $D\left(A^{\prime}\right)$ is a strongly free dendrite in $X$ with trunk $A^{\prime}$.

Lemma 2.5. Suppose $D_{1}$ and $D_{2}$ are two strongly free dendrites in the metric space $X$ with $A_{1}$ and $A_{2}$ being their respective trunks. If there is an arc $A \subseteq A_{1} \cap A_{2}$, then $D_{1}(A)=D_{2}(A)$.

Proof. First we show that $D_{1}(A) \subseteq D_{2}(A)$. Let $C$ be a component of $D_{1}(A)-A$ and $\bar{C} \cap \AA=\{x\}$. Since $D_{2}(A)-\operatorname{End}(A)$ is a component of $X-\operatorname{End}(A)$ and $\bar{C} \cap\left(D_{2}(A)-\operatorname{End}(A)\right) \neq \emptyset$, we have $\bar{C} \subseteq D_{2}(A)-\operatorname{End}(A)$. By the arbitrariness of $C, D_{1}(A)-A \subseteq D_{2}(A)$. Thus $D_{1}(A) \subseteq D_{2}(A)$.

Similarly, it can be shown that $D_{2}(A) \subseteq D_{1}(A)$.

The following lemma is obvious.

Lemma 2.6. Let $D$ be a strongly free dendrite in $X$ with trunk $A$, and let $h: X \rightarrow X$ be a homeomorphism. Then $h(D)$ is a strongly free dendrite in $X$ with the $\operatorname{arc} h(A)$ being its trunk. 
Let $X$ be a compact metric space and let $D$ be a strongly free dendrite in $X$ with trunk $A$. Define $\stackrel{\circ}{D}=D-\partial_{X}(D)=D-\operatorname{End}(A)$. Obviously $\stackrel{\circ}{D}$ is an open subset in $X$.

Lemma 2.7. Let $X$ be a Peano continuum, and let $D_{1}, \ldots, D_{n}$ be $n$ strongly free dendrites in $X$. If $\bigcup_{i=1}^{n} \stackrel{\circ}{D}_{i}$ is a connected open subset of $X$, then the boundary of $\bigcup_{i=1}^{n} \stackrel{\circ}{D}_{i}$ in $X$ has at most two points.

Proof. Without loss of generality, we may suppose that $\left(\bigcup_{i=1}^{k} \stackrel{\circ}{D}_{i}\right) \cap$ $\stackrel{\circ}{D}_{k+1} \neq \emptyset$ for each $k \in\{1, \ldots, n-1\}$. Let $A_{i}$ be the trunk of $D_{i}$ for each $i \in\{1, \ldots, n\}$. Now we argue by induction. Clearly $\partial_{X}\left(\stackrel{\circ}{D}_{1}\right)$ has only two points. Assume that $\partial_{X}\left(\bigcup_{i=1}^{k} \stackrel{\circ}{D}_{i}\right)$ has at most two points for some $1 \leq k<n$. We will show that $\partial_{X}\left(\bigcup_{i=1}^{k+1} \stackrel{\circ}{D}_{i}\right)$ has at most two points. Set $V=\bigcup_{i=1}^{k} \stackrel{\circ}{D}_{i}$. Since both $V$ and $\stackrel{\circ}{D}_{k+1}$ are open subsets of $X$, we have $\partial_{X}\left(V \cup \stackrel{\circ}{D}_{k+1}\right) \subseteq \partial_{X}(V) \cup \partial_{X}\left(\stackrel{\circ}{D}_{k+1}\right)$. Thus $\partial_{X}\left(V \cup \stackrel{\circ}{D}_{k+1}\right)$ has at most four points. There are three cases:

CASE 1: $\partial_{X}(V) \cap \stackrel{\circ}{D}_{k+1}=\emptyset$. Then $\stackrel{\circ}{D}_{k+1} \subseteq X-\partial_{X}(V)$. Since $V$ is a component of $X-\partial_{X}(V)$ and $\stackrel{\circ}{D}_{k+1} \cap V \neq \emptyset$, we have $\stackrel{\circ}{D}_{k+1} \subseteq V$. Thus $\partial_{X}\left(V \cup \stackrel{\circ}{D}_{k+1}\right)=\partial_{X}(V)$.

CASE 2: $\partial_{X}\left(\stackrel{\circ}{D}_{k+1}\right) \cap V=\emptyset$. Similarly to Case 1 , we have $V \subseteq \stackrel{\circ}{D}_{k+1}$. So $\partial_{X}\left(V \cup \stackrel{\circ}{D}_{k+1}\right)=\partial_{X}\left(\stackrel{\circ}{D}_{k+1}\right)=\operatorname{End}\left(A_{k+1}\right)$.

CASE 3: $\partial_{X}(V) \cap \stackrel{\circ}{D}_{k+1} \neq \emptyset$ and $\partial_{X}\left(\stackrel{\circ}{D}_{k+1}\right) \cap V \neq \emptyset$. Since no point of $\stackrel{\circ}{D}_{k+1}$ or $V$ can be in $\partial_{X}\left(V \cup \stackrel{\circ}{D}_{k+1}\right), \partial_{X}\left(V \cup \stackrel{\circ}{D}_{k+1}\right)$ has at most two points.

In any case, $\partial_{X}\left(\bigcup_{i=1}^{k+1} \stackrel{\circ}{D}_{i}\right)$ has at most two points. Thus the boundary of $\bigcup_{i=1}^{n} \stackrel{\circ}{D}_{i}$ in $X$ has at most two points by this inductive process.

The following lemma is well known (see [13, 8.30]).

Lemma 2.8. Let $X$ be a Peano continuum. Then for any $\varepsilon>0$, there is $a \delta=\delta(\varepsilon) \in(0, \varepsilon / 2]$ such that, for any $x, y \in X$ with $0<d(x, y) \leq \delta$, there always exists an arc $A$ in $X$ with endpoints $x$ and $y$ and $\operatorname{diam}(A)<\varepsilon$.

Let $A$ be an arc, and let $\prec$ be an ordering in $A$. If there is a homeomorphism $h: A \rightarrow I(=[0,1])$ such that, for any $x, y \in A, x \prec y$ if and only if $h(x)<h(y)$, then $\prec$ is called a natural ordering. Suppose that $y_{0}, y_{1}, \ldots, y_{m}$ are points in $A, m \geq 2$, and $\operatorname{End}(A)=\left\{y_{0}, y_{m}\right\}$. If $y_{0} \prec y_{1} \prec \cdots \prec y_{m}$ in a natural ordering $\prec$ in $A$, and $d\left(y_{k}, y_{0}\right)=k d\left(y_{m}, y_{0}\right) / m$ for all $k=1, \ldots, m$, then the sequence $\left(y_{1}, \ldots, y_{m-1}\right)$ is said to be a set of pseudo m-section points of $A$ from $y_{0}$ to $y_{m}$.

Let $A$ be an arc. For any two different points $u$ and $v$ in $A,[u, v]_{A}$ denotes the subarc of $A$ with endpoints $u$ and $v$. Write $[u, v)_{A}=(v, u]_{A}=$ $[u, v]_{A}-\{v\},(u, v)_{A}=[u, v)_{A}-\{u\}$, and $[u, u]_{A}=\{u\}$. 
Lemma 2.9. Let $\left\{D_{n}: n=1,2, \ldots\right\}$ be a sequence of strongly free dendrites in a Peano continuum $X$. Suppose that $\bigcup_{n=1}^{\infty} \stackrel{\circ}{D}_{n}$ is connected and contains no simple closed curve. If $\left\{A_{i}: i=1,2, \ldots\right\}$ is a sequence of pairwise disjoint closed arcs in $\bigcup_{n=1}^{\infty} \stackrel{\circ}{D}_{n}$, then $\lim _{i \rightarrow \infty} \operatorname{diam}\left(A_{i}\right)=0$.

Proof. Since $\bigcup_{n=1}^{\infty} \stackrel{\circ}{D}_{n}$ is connected, by relabeling the $D_{n}$ we may suppose that $\stackrel{\circ}{D}_{k+1} \cap \bigcup_{n=1}^{k} \stackrel{\circ}{D}_{n} \neq \emptyset$ for each $k \in \mathbb{N}$.

Assume to the contrary that there exist a constant $c>0$ and infinitely many $A_{i}$ such that $\operatorname{diam}\left(A_{i}\right) \geq c$. We can suppose that each $A_{i}$ has $\operatorname{diam}\left(A_{i}\right)=d\left(a_{i}, b_{i}\right)=c$, where $a_{i}$ and $b_{i}$ are the endpoints of $A_{i}$. For each $i$, let $y_{i 0}=a_{i}, y_{i 5}=b_{i}$ and let $\left(y_{i 1}, \ldots, y_{i 4}\right)$ be a set of pseudo 5 -section points of $A_{i}$ from $y_{i 0}$ to $y_{i 5}$. Then $\operatorname{diam}\left(\left[y_{i k}, y_{i k+1}\right]_{A_{i}}\right) \geq c / 5$ for each $k=0,1, \ldots, 4$. Let $\varepsilon=c / 10$, and let $\delta=\delta(\varepsilon) \in(0, \varepsilon / 2]$ be as in Lemma 2.8.

As $X$ is compact, there are integers $q>p>0$ such that $\max \left\{d\left(y_{p k}, y_{q k}\right)\right.$ : $k=0,1,2,3,4,5\}<\delta$. By Lemma 2.8 , there is an arc $L_{k}$ from $y_{p k}$ to $y_{q k}$ such that $\operatorname{diam}\left(L_{k}\right)<c / 10$ for each $k \in\{0, \ldots, 5\}$. It is easy to see that $\left\{L_{k}: k=\right.$ $0, \ldots, 5\}$ are pairwise disjoint. (If there is some $x \in L_{k} \cap L_{j}$, then $d\left(y_{p k}, y_{p j}\right) \leq$ $d\left(y_{p k}, x\right)+d\left(x, y_{p j}\right) \leq \operatorname{diam}\left(L_{k}\right)+\operatorname{diam}\left(L_{j}\right)<c / 5$, a contradiction.) Since the compact set $A_{p} \cup A_{q}$ is covered by the family of open sets $\left\{\stackrel{\circ}{D}_{n}: n=\right.$ $1,2, \ldots\}$, there exists an $N$ such that $A_{p} \cup A_{q} \subset \bigcup_{n=1}^{N} \stackrel{\circ}{D}_{n}$. By Lemma 2.7, the boundary of $\bigcup_{n=1}^{N} \stackrel{\circ}{D}_{n}$ in $X$ has at most two points. So, at most two arcs among $\left\{L_{k}: k=0, \ldots, 5\right\}$ contain points in $\partial_{X}\left(\bigcup_{n=1}^{N} \stackrel{\circ}{D}_{n}\right)$. Thus there are distinct $\operatorname{arcs} L_{l}$ and $L_{m}$ such that $\left(L_{l} \cup L_{m}\right) \cap \partial_{X}\left(\bigcup_{n=1}^{N} \stackrel{\circ}{D}_{n}\right)=\emptyset$. Then $L_{l}, L_{m} \subset \bigcup_{n=1}^{N} \stackrel{\circ}{D}_{n}$. Let $v_{l} \in L_{l} \cap A_{p}$ be such that $\left[y_{q l}, v_{l}\right]_{L_{l}} \cap A_{p}=\left\{v_{l}\right\}$, and $v_{m} \in L_{m} \cap A_{p}$ be such that $\left[y_{q m}, v_{m}\right]_{L_{m}} \cap A_{p}=\left\{v_{m}\right\}$. Since both $\left[y_{q l}, v_{l}\right]_{L_{l}} \cup$ $\left[v_{l}, v_{m}\right]_{A_{p}} \cup\left[v_{m}, y_{q m}\right]_{L_{m}}$ and $\left[y_{q l}, y_{q m}\right]_{A_{q}}$ are arcs from $y_{q l}$ to $y_{q m}$, and $\bigcup_{n=1}^{N} \stackrel{\circ}{D}_{n}$ has no simple closed curve, we have $\left[y_{q l}, v_{l}\right]_{L_{l}} \cup\left[v_{l}, v_{m}\right]_{A_{p}} \cup\left[v_{m}, y_{q m}\right]_{L_{m}}=$ $\left[y_{q l}, y_{q m}\right]_{A_{q}}$. Thus $\left[v_{l}, v_{m}\right]_{A_{p}} \subset\left[y_{q l}, y_{q m}\right]_{A_{q}}$, contrary to $A_{p} \cap A_{q}=\emptyset$.

Lemma $2.10([13,8.26])$. Any connected open subset of a Peano continuum is arcwise connected.

If $X$ is a topological space and $A, B, C \subset X$, then we say that $C$ separates $A$ and $B$ in $X$ provided that $X-C=P \cup Q$ with $A \subset P$ and $B \subset Q$, where $P$ and $Q$ are disjoint nonempty open subsets of $X-C$. If $x, y, z \in X$, we usually say that $z$ separates $x$ and $y$ in $X$ instead of saying that $\{z\}$ separates $\{x\}$ and $\{y\}$ in $X$.

Lemma 2.11. Let $X$ be a Peano continuum. Suppose $U$ is a connected open subset of $X$ and contains no simple closed curve. Then any two points of $U$ are separated in $U$ by a third point of $U$.

Proof. Let $p, q \in U$ and $p \neq q$. By Lemma 2.10, $U$ is arcwise connected. So there is an $\operatorname{arc} A$ in $U$ from $p$ to $q$. Let $r \in A-\{p, q\}$, and let $V$ be the 
component of $p$ in $U-\{r\}$. Assume that $p$ and $q$ cannot be separated in $U$ by $r$. Then $q \in V$. Since $X$ is locally connected, the component $V$ is open in $U-\{r\}$. Thus $V$ is open in $X$. By Lemma 2.10 there is an arc $B$ in $V$ from $p$ to $q$. Clearly, $A \cap B$ is not connected, so $A \cup B$ contains a simple closed curve. This contradicts the assumption that $U$ contains no simple closed curve. Thus $p$ and $q$ are separated in $U$ by $r$.

Proposition 2.12. Let $\left\{D_{n}: n=1,2, \ldots\right\}$ be a sequence of strongly free dendrites in a Peano continuum $X$. Suppose that $\bigcup_{n=1}^{\infty} \stackrel{\circ}{D}_{n}$ is connected and has no simple closed curve. Then for any constant $c>0$, there exists a finite set $\left\{x_{1}, \ldots, x_{m}\right\} \subset \bigcup_{n=1}^{\infty} \stackrel{\circ}{D}_{n}$ such that each component of $\left(\bigcup_{n=1}^{\infty} \stackrel{\circ}{D}_{n}\right)$ $\left\{x_{1}, \ldots, x_{m}\right\}$ has diameter $\leq c$.

Proof. Assume to the contrary that for any finite set $S \subset \bigcup_{n=1}^{\infty} \stackrel{\circ}{D}_{n}$, there exists a component of $\left(\bigcup_{n=1}^{\infty} \stackrel{\circ}{D}_{n}\right)-S$ with diameter $>c$. Write $E=$ $\bigcup_{n=1}^{\infty} \stackrel{\circ}{D}_{n}$. By Lemma 2.11, we can choose $x_{1} \in E$ such that $E-\left\{x_{1}\right\}$ has at least two components. Then there is a component $U_{1}$ of $E-\left\{x_{1}\right\}$ with $\operatorname{diam}\left(U_{1}\right)>c$. Take an $\operatorname{arc} L_{1} \subset U_{1}$ such that $\operatorname{diam}\left(L_{1}\right)=c$. Choose $x_{2}, x_{3} \in$ $\stackrel{\circ}{1}_{1}$ with $\operatorname{diam}\left(\left[x_{2}, x_{3}\right]_{L_{1}}\right)=c / 4$. By the proof of Lemma 2.11 , it is easy to see that $x_{2}, x_{3} \in \operatorname{Cut}\left(U_{1}\right)$. In $E-\left\{x_{1}, x_{2}, x_{3}\right\}$, there is also a component $U_{2}$ with $\operatorname{diam}\left(U_{2}\right)>c$. Take an $\operatorname{arc} L_{2}$ in $U_{2}$ such that $\operatorname{diam}\left(L_{2}\right)=c$. If $L_{2} \cap\left[x_{2}, x_{3}\right]_{L_{1}}=\emptyset$, then we choose arbitrarily a subarc $\left[x_{4}, x_{5}\right]_{L_{2}} \subset \stackrel{\circ}{L}_{2}$ with $\operatorname{diam}\left(\left[x_{4}, x_{5}\right]_{L_{2}}\right)=c / 4$. Otherwise, $L_{2} \cap\left[x_{2}, x_{3}\right]_{L_{1}} \neq \emptyset$. Since there is no simple closed curve in $E$, the intersection $A=L_{2} \cap\left[x_{2}, x_{3}\right]_{L_{1}}$ must be an arc with diameter $\leq c / 4$. Thus $L_{2}-A$ has a subarc with diameter $>c / 4$. Select $x_{4}, x_{5} \in \operatorname{Int}\left(L_{2}-A\right)$ such that $\left[x_{4}, x_{5}\right]_{L_{2}} \subset L_{2}-A$ and $\operatorname{diam}\left(\left[x_{4}, x_{5}\right]_{L_{2}}\right)=c / 4$.

Suppose that $\left\{x_{1}, \ldots, x_{2 k+1}\right\}$ and $\left\{L_{1}, \ldots, L_{k}\right\}$ have been chosen such that

(1) for each $i=1, \ldots, k, L_{i}$ is an arc in $E-\left\{x_{1}, \ldots, x_{2 i-1}\right\}$ and $x_{2 i}, x_{2 i+1}$ $\in \stackrel{\circ}{L}_{i}$;

(2) for each $i=1, \ldots, k, \operatorname{diam}\left(\left[x_{2 i}, x_{2 i+1}\right]_{L_{i}}\right)=c / 4$;

(3) for each $i, j=1, \ldots, k$ with $i \neq j,\left[x_{2 i}, x_{2 i+1}\right]_{L_{i}} \cap\left[x_{2 j}, x_{2 j+1}\right]_{L_{j}}=\emptyset$;

(4) for each $i, j=1, \ldots, k$ with $i \neq j,\left(x_{2 i}, x_{2 i+1}\right)_{L_{i}}$ and $\left(x_{2 j}, x_{2 j+1}\right)_{L_{j}}$ lie in different components of $E-\left\{x_{1}, \ldots, x_{2 k+1}\right\}$.

For $k+1$, there is also a component $U_{k+1}$ in $E-\left\{x_{1}, \ldots, x_{2 k+1}\right\}$ with $\operatorname{diam}\left(U_{k+1}\right)>c$ by assumption. Take an arc $L_{k+1} \subset U_{k+1}$ with $\operatorname{diam}\left(L_{k+1}\right)$ $=c$. If $L_{k+1} \cap\left[x_{2 i}, x_{2 i+1}\right]_{L_{i}}=\emptyset$ for all $i \in\{1, \ldots, k\}$, then we choose any subarc $\left[x_{2 k+2}, x_{2 k+3}\right]_{L_{k+1}} \subset \stackrel{\circ}{L_{k+1}}$ with $\operatorname{diam}\left(\left[x_{2 k+2}, x_{2 k+3}\right]_{L_{k+1}}\right)=c / 4$. Otherwise, according to (4), there is only one arc $\left[x_{2 i}, x_{2 i+1}\right]_{L_{i}}$ which has a nonempty intersection with $L_{k+1}$. Since there is no simple closed curve in $E$, the intersection $A=L_{k+1} \cap\left[x_{2 i}, x_{2 i+1}\right]_{L_{i}}$ must be an arc with diameter $\leq c / 4$. Thus $L_{k+1}-A$ has a subarc with diameter $>c / 4$. Select 
$x_{2 k+2}, x_{2 k+3} \in \operatorname{Int}\left(L_{k+1}-A\right)$ such that $\left[x_{2 k+2}, x_{2 k+3}\right]_{L_{k+1}} \subset L_{k+1}-A$ and $\operatorname{diam}\left(\left[x_{2 k+2}, x_{2 k+3}\right]_{L_{k+1}}\right)=c / 4$.

Inductively, we obtain a sequence $\left\{\left[x_{2 i}, x_{2 i+1}\right]_{L_{i}}\right\}_{i=1}^{\infty}$ of pairwise disjoint $\operatorname{arcs}$ in $E$ with $\operatorname{diam}\left(\left[x_{2 i}, x_{2 i+1}\right]_{L_{i}}\right)=c / 4$. This contradicts Lemma 2.9.

Lemma 2.13. Let $\left\{D_{n}: n=1,2, \ldots\right\}$ be a sequence of strongly free dendrites in a Peano continuum $X$. Suppose $\bigcup_{n=1}^{\infty} \stackrel{\circ}{D}_{n}=U_{1} \cup U_{2} \cup \cdots$, where $\left\{U_{i}: i=1,2, \ldots\right\}$ is a sequence of pairwise disjoint connected open sets. Then $\lim _{i \rightarrow \infty} \operatorname{diam}\left(U_{i}\right)=0$.

Proof. Assume that there exist a constant $c>0$ and infinitely many $U_{i}$ such that $\operatorname{diam}\left(U_{i}\right)>c$. We may suppose that $\operatorname{diam}\left(U_{i}\right)>c$ for each $i=1,2, \ldots$. Choose an arc $A_{i} \subset U_{i}$ such that $\operatorname{diam}\left(A_{i}\right)=d\left(a_{i}, b_{i}\right)=c$, where $a_{i}$ and $b_{i}$ are the endpoints of $A_{i}$. For each $i=1,2, \ldots$, let $y_{i 0}=a_{i}$, $y_{i 5}=b_{i}$ and let $\left(y_{i 1}, \ldots, y_{i 4}\right)$ be a set of pseudo 5 -section points of $A_{i}$ from $y_{i 0}$ to $y_{i 5}$. Then $\operatorname{diam}\left(\left[y_{i k}, y_{i k+1}\right]\right) \geq c / 5$ for each $k=0,1, \ldots, 4$. Let $\varepsilon=c / 10$ and let $\delta=\delta(\varepsilon) \in(0, \varepsilon / 2]$ be as in Lemma 2.8.

As $X$ is compact, there are $n>m>0$ such that $\max \left\{d\left(y_{m k}, y_{n k}\right): k=\right.$ $0, \ldots, 5\} \leq \delta$. By Lemma 2.8, there exists an arc $L_{k}$ from $y_{m k}$ to $y_{n k}$ with $\operatorname{diam}\left(L_{k}\right)<\varepsilon=c / 10$ for each $k=0, \ldots, 5$. Clearly $\left\{L_{k}: k=0, \ldots, 5\right\}$ are pairwise disjoint.

By the compactness of $A_{m}$, there exist integers $m_{1}, \ldots, m_{N}$ such that $A_{m} \subset \bigcup_{j=1}^{N} \stackrel{\circ}{D}_{m_{j}} \subseteq U_{m}$ and $\bigcup_{j=1}^{N} \stackrel{\circ}{D}_{m_{j}}$ is connected. By Lemma 2.7, $\partial_{X}\left(\bigcup_{j=1}^{N} \stackrel{\circ}{D}_{m_{j}}\right)$ has at most two points. Hence, there is some $L_{k}$ such that $L_{k} \cap \partial_{X}\left(\bigcup_{j=1}^{N} \stackrel{\circ}{D}_{m_{j}}\right)=\emptyset$. Thus $L_{k} \subset \bigcup_{j=1}^{N} \stackrel{\circ}{D}_{m_{j}} \subseteq U_{m}$. So $y_{n k} \in U_{m}$. This is a contradiction.

Proposition 2.14. Let $\left\{D_{n}: n=1,2, \ldots\right\}$ be a sequence of strongly free dendrites in a Peano continuum $X$. If $\bigcup_{n=1}^{\infty} D_{n}$ contains no simple closed curve, then for any constant $c>0$, there exists a finite set $\left\{x_{1}, \ldots, x_{m}\right\} \subset$ $\bigcup_{n=1}^{\infty} \stackrel{\circ}{D}_{n}$ such that each component of $\left(\bigcup_{n=1}^{\infty} \stackrel{\circ}{D}_{n}\right)-\left\{x_{1}, \ldots, x_{m}\right\}$ has diameter $\leq c$.

Proof. Write $\bigcup_{n=1}^{\infty} \stackrel{\circ}{D}_{n}=U_{1} \cup U_{2} \cup \cdots$, where $\left\{U_{i}\right\}_{i=1}^{\infty}$ are pairwise disjoint open connected subsets. (If $\bigcup_{n=1}^{\infty} \stackrel{\circ}{D}_{n}$ has only finitely many components, that is, $\cup_{n=1}^{\infty} \stackrel{\circ}{D}_{n}=U_{1} \cup \cdots \cup U_{M}$, then let $U_{i}=\emptyset$ for $i>M$. Thus we can still write $\bigcup_{n=1}^{\infty} \stackrel{\circ}{D}_{n}=U_{1} \cup U_{2} \cup \cdots$.) Because of Lemma 2.13, $\operatorname{diam}\left(U_{i}\right) \rightarrow 0$ as $i \rightarrow \infty$. Then for any $c>0$ there exists an $N$ such that $\operatorname{diam}\left(U_{i}\right) \leq c$ for $i>N$. For each $i \leq N$, since $U_{i}$ is the union of some $\stackrel{\circ}{D}_{n} \mathrm{~s}$, there is a finite set $F_{i} \subset U_{i}$ such that each component of $U_{i}-F_{i}$ has diameter $\leq c$ by Proposition 2.12. Thus each component of $\left(\bigcup_{n=1}^{\infty} \stackrel{\circ}{n}_{n}\right)-\left(\bigcup_{i=1}^{N} F_{i}\right)$ has diameter $\leq c$. Taking $\left\{x_{1}, \ldots, x_{m}\right\}=\bigcup_{i=1}^{N} F_{i}$ completes the proof. 
Corollary 2.15. Let $X$ be a Peano continuum, and $D$ a strongly free dendrite in $X$. Suppose that $G$ is a countable group acting on $X$. If $\bigcup_{g \in G} g \stackrel{\circ}{D}$ has no simple closed curve, then for any constant $c>0$, there exists a finite set $\left\{x_{1}, \ldots, x_{m}\right\}$ such that each component of $\left(\bigcup_{g \in G} g \stackrel{\circ}{D}\right)-\left\{x_{1}, \ldots, x_{m}\right\}$ has diameter $\leq c$.

Proof. From the definition of strongly free dendrite and Lemma 2.6, it is easy to see that $g D$ is also a strongly free dendrite and $(g \stackrel{\circ}{D})=g \stackrel{\circ}{D}$ for each $g \in G$. Hence, the conclusion follows immediately from Proposition 2.14.

REMARK. In fact, Corollary 2.15 holds for any subset of $\bigcup_{g \in G} g \stackrel{\circ}{D}$. Let $W \subseteq \bigcup_{g \in G} g \stackrel{\circ}{D}$, and $\left\{x_{1}, \ldots, x_{m}\right\} \subset \bigcup_{g \in G} g \stackrel{\circ}{D}$. If each component of $\left(\bigcup_{g \in G} g \stackrel{\circ}{D}\right)-\left\{x_{1}, \ldots, x_{m}\right\}$ has diameter $\leq c$, we can take the finite set $F=$ $W \cap\left\{x_{1}, \ldots, x_{m}\right\}$. Obviously, each component of $W-F$ has diameter $\leq c$.

The action of $G$ on $X$ is said to be topologically transitive if for any nonempty open subsets $U$ and $V$ of $X$, there is a $g \in G$ such that $g U \cap V \neq \emptyset$. For brevity, we will then simply say that $G$ is topologically transitive.

We say $x \in X$ is a transitive point of $G$ provided that $\overline{G x}=X$. For a compact metric space $X$, it is well known that if $G$ is countable and topologically transitive, then there exists a transitive point of $G$.

Proposition 2.16. Let $X$ be a Peano continuum containing free dendrites, and $D$ a strongly free dendrite in $X$. Suppose that $G$ is a countable group acting on $X$ expansively. If $\bigcup_{g \in G} g \stackrel{\circ}{D}$ contains no simple closed curve, then there is a $G$-invariant open set $W$ on which $\left.G\right|_{W}$ is topologically transitive.

Proof. Let $c_{0}>0$ be an expansive constant for the $G$-action. Set $U=$ $\bigcup_{g \in G} g \stackrel{\circ}{D}$. Then $U$ is a $G$-invariant open set. By Corollary 2.15, there is a finite set $\left\{x_{1}, \ldots, x_{m}\right\} \subset U$ such that each component of $U-\left\{x_{1}, \ldots, x_{m}\right\}$ has diameter $\leq c_{0}$.

Claim. There exists an $x_{i} \in\left\{x_{1}, \ldots, x_{m}\right\}$ with $\operatorname{Int}\left(\overline{G x_{i}}\right) \neq \emptyset$.

Proof of the claim. Assume that $\operatorname{Int}\left(\overline{G x_{i}}\right)=\emptyset$ for each $i \in\{1, \ldots, m\}$. Since $U$ is a nonempty open set, so is $V:=U-\bigcup_{i=1}^{m} \overline{G x_{i}}$ by the Baire Category Theorem. Thus we can take distinct points $x$ and $y$ in some component of $V$. Since $V$ is $G$-invariant, for any $g \in G, g x$ and $g y$ are still in the same component of $V$. As $V \subset U-\left\{x_{1}, \ldots, x_{m}\right\}$, each component of $V$ has diameter $\leq c_{0}$. Hence $d(g x, g y) \leq c_{0}$ for any $g \in G$, which contradicts the fact that $G$ acts on $X$ expansively. Thus the claim holds.

Without loss of generality, we suppose $\operatorname{Int}\left(\overline{G x_{1}}\right) \neq \emptyset$. Let $W=U \cap$ $\operatorname{Int}\left(\overline{G x_{1}}\right)$. Obviously, $W$ is a $G$-invariant open set. To prove that $\left.G\right|_{W}$ is topologically transitive, let $A$ and $B$ be nonempty open subsets of $W$. Since 
$A, B \subseteq \operatorname{Int}\left(\overline{G x_{1}}\right)$, there are $g_{1}, g_{2} \in G$ such that $g_{1} x_{1} \in A$ and $g_{2} x_{1} \in B$. So $g_{2} g_{1}^{-1}\left(g_{1} x_{1}\right) \in B$, that is, $g_{2} g_{1}^{-1} A \cap B \neq \emptyset$. Hence $\left.G\right|_{W}$ is topologically transitive.

Proposition 2.17. Under the assumption of Proposition 2.16, let $W$ be a $G$-invariant open set in $\bigcup_{g \in G} g \stackrel{\circ}{D}$ such that $\left.G\right|_{W}$ is topologically transitive. Then there is an arc $A$ in $W$ such that each point in $A$ is a transitive point of $\left.G\right|_{W}$.

Proof. Assume that on any arc in $W$, there exists a nontransitive point of $\left.G\right|_{W}$. Then we have

Claim. For any constant $c>0$, there is a finite subset $B \subseteq W$ consisting of nontransitive points of $\left.G\right|_{W}$ such that each component of $W-B$ has diameter $\leq c$.

Proof of the claim. As in Proposition 2.16, we set $U=\bigcup_{g \in G} g \stackrel{\circ}{D}$. By Corollary 2.15 and the remark following it, there is a finite set $\left\{x_{1}, \ldots, x_{M}\right\}$ $\subseteq W$ such that each component of $W-\left\{x_{1}, \ldots, x_{M}\right\}$ has diameter $\leq c$. If $x_{i}$ is a nontransitive point of $\left.G\right|_{W}$, then take $B=\left\{x_{1}, \ldots, x_{M}\right\}$. Otherwise, we will modify the set $\left\{x_{1}, \ldots, x_{M}\right\}$. By renumbering the $x_{i}$, we may suppose that $x_{1}, \ldots, x_{m-1}$ are nontransitive points of $\left.G\right|_{W}$ and $x_{m}, \ldots, x_{M}$ are transitive points of $\left.G\right|_{W}$, for some $1 \leq m \leq M$. Then we first modify the point $x_{m}$.

Let $\mathcal{P}$ be family of components $P$ of $W-\left\{x_{1}, \ldots, x_{M}\right\}$ such that $x_{m} \in$ $\partial_{X}(P)$. Write $\mathcal{P}=\left\{P_{1}, P_{2}, \ldots\right\}$ (if $\mathcal{P}$ has only finitely many elements, then set $P_{n}=\emptyset$ for $n>N$, for some $\left.N \in \mathbb{N}\right)$. Then $\operatorname{diam}\left(P_{n}\right) \rightarrow 0$ as $n \rightarrow 0$. (Otherwise, for a constant $\varepsilon>0$, there is a subsequence $\left\{P_{n_{k}}\right\}_{k=1}^{\infty}$ such that each $\operatorname{diam}\left(P_{n_{k}}\right)>\varepsilon$. Since $P_{n_{k}}$ is arcwise connected, there is an arc $L_{n_{k}}$ with $\operatorname{diam}\left(L_{n_{k}}\right)=\varepsilon$. This contradicts Lemma 2.9.) Thus there exists an integer $N>0$ such that $\operatorname{diam}\left(P_{n}\right)<c / 4$ for each $n>N$. Let $P_{n}^{\prime}=P_{n} \cup\left\{x_{m}\right\}$ for each $n \in \mathbb{N}$. Then for any distinct $x, y \in \bigcup_{n=N+1}^{\infty} P_{n}^{\prime}$, we have $d(x, y) \leq$ $d\left(x, x_{m}\right)+d\left(x_{m}, y\right)<c / 2$. Thus $\operatorname{diam}\left(\bigcup_{n=N+1}^{\infty} P_{n}^{\prime}\right) \leq c / 2$.

For each $n \in\{1, \ldots, N\}$, let $L_{n}$ be an arc in $P_{n}^{\prime}$ with $x_{m}$ being one of its endpoints and $\operatorname{diam}\left(L_{n}\right)<c / 4$. By assumption there exists a nontransitive point $y_{n} \in \stackrel{\circ}{L}_{n}$ of $\left.G\right|_{W}$ such that the component of $P_{n}^{\prime}-\left\{y_{n}\right\}$ which contains $x_{m}$ has diameter $\leq c / 2$. In fact, for each $x \in P_{n}$ there is a unique arc $A_{x}=\left[x, a_{x}\right]$ contained in $P_{n}$ such that $A_{x} \cap L_{n}=\left\{a_{x}\right\}$. Choose a nontransitive point $w_{1} \in \stackrel{\circ}{L}_{n}$ such that $\operatorname{diam}\left(\left[x_{m}, w_{1}\right]_{L_{n}}\right) \leq c / 6$. If the component of $P_{n}^{\prime}-\left\{w_{1}\right\}$ which contains $x_{m}$ has diameter $\leq c / 2$, then let $y_{n}=w_{1}$. Otherwise, there must be a point $z_{1} \in P_{n}$ and an arc $A_{z_{1}}=\left[z_{1}, a_{z_{1}}\right]$ such that $\operatorname{diam}\left(A_{z_{1}}\right)>c / 6$ and $A_{z_{1}} \cap\left(x_{m}, w_{1}\right)_{L_{n}}=\left\{a_{z_{1}}\right\}$. Then choose a nontransitive point $w_{2} \in\left(x_{m}, a_{z_{1}}\right)_{L_{n}}$. If the component of $P_{n}^{\prime}-\left\{w_{2}\right\}$ which contains $x_{m}$ has diameter $\leq c / 2$, then let $y_{n}=w_{2}$. Otherwise, there is a 
$z_{2}$ with $\operatorname{diam}\left(A_{z_{2}}\right)>c / 6$. Continuing this process, we get at last a $w_{k}$ such that the component of $P_{n}^{\prime}-\left\{w_{k}\right\}$ which contains $x_{m}$ has diameter $\leq c / 2$, and then we let $y_{n}=w_{k}$. (If such a $w_{k}$ does not exist, then we will obtain a sequence $\left\{A_{z_{i}}\right\}_{i=1}^{\infty}$ of pairwise disjoint $\operatorname{arcs}$ with $\operatorname{diam}\left(A_{z_{i}}\right)>c / 6$, contradicting Lemma 2.9.)

Thus for each $P_{n}^{\prime}, n=1, \ldots, N$, we get a nontransitive point $y_{n}$. Since each component of $P_{n}^{\prime}-\left\{y_{n}\right\}$ which does not contain $x_{m}$ is contained in $P_{n}$, its diameter is at most $c$. Let $C_{n}$ denote the component of $P_{n}^{\prime}-\left\{y_{n}\right\}$ which contains $x_{m}$. Since $x_{m} \in C_{n}$ and $x_{m} \in \bigcup_{n=N+1}^{\infty} P_{n}^{\prime}$, the set $\left(\bigcup_{n=1}^{N} C_{n}\right) \cup$ $\left(\bigcup_{n=N+1}^{\infty} P_{n}^{\prime}\right)$ is connected and it is easy to see that its diameter is at most $c$. Thus using $\left\{y_{1}, \ldots, y_{N}\right\}$ instead of $x_{m}$, we obtain a new finite set $B^{\prime}=\left\{x_{1}, \ldots, x_{m-1}, y_{1}, \ldots, y_{N}, x_{m+1}, \ldots, x_{M}\right\}$ such that each component of $W-B^{\prime}$ has diameter $\leq c$.

Similarly, we can modify $x_{m+1}, \ldots, x_{M}$ by using nontransitive points. Finally, we obtain a finite set $B$ we need. Thus the claim is proved.

Let $c_{0}$ be an expansive constant for the $G$-action. From the claim, we get a finite set $\left\{x_{1}, \ldots, x_{n}\right\}$ of nontransitive points such that each component of $W-\left\{x_{1}, \ldots, x_{n}\right\}$ has diameter $\leq c_{0}$. For each $x_{i}$, Int $\left(\overline{G x_{i}}\right)=\emptyset$ since $x_{i}$ is a nontransitive point of $\left.G\right|_{W}$. Thus $V=W-\bigcup_{i=1}^{n} \overline{G x_{i}}$ is a nonempty $G$-invariant open set. Obviously, each component of $V$ has diameter $\leq c_{0}$. This contradicts the fact that $G$ acts on $X$ expansively with expansive constant $c_{0}$.

Proposition 2.18. Let $X$ be a Peano continuum, and $D_{1}, \ldots, D_{n}$ a finite sequence of strongly free dendrites in $X$. If $\bigcup_{i=1}^{n} \stackrel{\circ}{D}_{i}$ contains a simple closed curve, then $X=\bigcup_{i=1}^{n} \stackrel{\circ}{D}_{i}$. Moveover, there is only one simple closed curve contained in $X$.

Proof. Let $S$ be a simple closed curve contained in $\bigcup_{i=1}^{n} \stackrel{\circ}{D}_{i}$. We may suppose that the union of any $n-1$ of $\stackrel{\circ}{D}_{1}, \ldots, \stackrel{\circ}{D}_{n}$ contains no simple closed curve (otherwise we need only work with a subfamily of $\left\{D_{1}, \ldots, D_{n}\right\}$ ). Thus $S \cap \stackrel{\circ}{D}_{i} \neq \emptyset$. By relabeling, we may suppose that $\bigcup_{i=1}^{n-1} \stackrel{\circ}{D}_{i}$ is connected and set $U=\bigcup_{i=1}^{n-1} \stackrel{\circ}{D}_{i}$. Let $\partial_{X}\left(\stackrel{\circ}{D}_{n}\right)=\{p, q\}$. By Lemma 2.7 the boundary of $U$ has at most two points.

First, it is easy to see that $\partial_{X}(U) \neq \emptyset$ : otherwise, $U$ is a clopen set in $X$, so $X=U$ by the connectedness of $X$. Then $S \subseteq \bigcup_{i=1}^{n} \stackrel{\circ}{D}_{i} \subseteq U$, which contradicts the fact that $U$ contains no simple closed curve.

Now we discuss two cases.

CASE 1: $\partial_{X}(U)=\{a\}$, that is, $\partial_{X}(U)$ has only one point. We claim that $\partial_{X}(U) \cap S \neq \emptyset$ and $\partial_{X}\left(\stackrel{D}{D}_{n}\right) \cap S \neq \emptyset$. Indeed, if $\partial_{X}(U) \cap S=\emptyset$, then $S \subset X-\partial_{X}(U)$. Since $U$ is a component of $X-\partial_{X}(U)$ and $S \cap U \neq \emptyset$, we find that $S \subset U$, which contradicts the fact that $U$ contains no simple closed 
curve. Hence $\partial_{X}(U) \cap S \neq \emptyset$. Similarly, it can be shown that $\partial_{X}\left(\stackrel{\circ}{D}_{n}\right) \cap S \neq \emptyset$. Thus $a \in S$. We can suppose $p \in S$. If $q \notin S$, then $S-\{p\}=S-\{p, q\} \subset$ $X-\{p, q\}$. Since $\stackrel{\circ}{D}_{n}$ is a component of $X-\{p, q\}$ and $(S-\{p\}) \cap \stackrel{\circ}{D}_{n} \neq \emptyset$, we have $S-\{p\} \subset \stackrel{\circ}{D}_{n}$. Thus $S \subset D_{n}$, a contradiction. Hence $q \in S$. It follows that $\partial_{X}\left(\bigcup_{i=1}^{n} \stackrel{\circ}{D}_{i}\right) \subseteq \partial_{X}(U) \cup \partial_{X}\left(\stackrel{\circ}{D}_{n}\right) \subseteq S \subseteq \bigcup_{i=1}^{n} \stackrel{\circ}{D}_{i}$. This means $\bigcup_{i=1}^{n} \stackrel{\circ}{D}_{i}$ is a clopen set in $X$. Hence $X=\bigcup_{i=1}^{n} \stackrel{\circ}{D}_{i}$.

CASE 2: $\partial_{X}(U)=\{a, b\}$, that is, $\partial_{X}(U)$ has two points. As in Case 1, we have $\partial_{X}(U) \cap S \neq \emptyset$ and $\partial_{X}\left(\stackrel{\circ}{D}_{n}\right) \cap S \neq \emptyset$. We can suppose that $a, p \in S$. Now we show that $b, q \in S$. First, we have $q \in S$ as in Case 1 . Assume to the contrary that $b \notin S$. As $\partial_{X}(U) \subseteq \bigcup_{i=1}^{n-1} \partial_{X}\left(\stackrel{\circ}{D}_{i}\right)$, there is some $\stackrel{\circ}{D}_{j}$ such that $b \in \partial_{X}\left(\stackrel{\circ}{D}_{j}\right)$. Let $\partial_{X}\left(\stackrel{\circ}{D}_{j}\right)=\{b, c\}$. Since $b \notin S, S-\partial_{X}\left(\stackrel{\circ}{D}_{j}\right)=S-\{c\}$ is connected. As $\left(S-\partial_{X}\left(\stackrel{\circ}{D}_{j}\right)\right) \cap \stackrel{\circ}{D}_{j} \neq \emptyset$ and $\stackrel{\circ}{D}_{j}$ is a component of $X-\partial_{X}\left(\stackrel{\circ}{D}_{j}\right)$, we have $S-\partial_{X}\left(\stackrel{\circ}{D}_{j}\right) \subseteq \stackrel{\circ}{D}_{j}$. So $S \subseteq D_{j}$, which is a contradiction. Hence $b \in S$. Thus $\partial_{X}\left(\bigcup_{i=1}^{n} \stackrel{\circ}{D}_{i}\right) \subseteq \partial_{X}(U) \cup \partial_{X}\left(\stackrel{\circ}{D}_{n}\right) \subseteq S \subseteq \bigcup_{i=1}^{n} \stackrel{\circ}{D}_{i}$. So $\bigcup_{i=1}^{n} \stackrel{\circ}{D}_{i}$ is a clopen set. Then $X=\bigcup_{i=1}^{n} \stackrel{\circ}{D}_{i}$.

Next we show that $X$ contains only one simple closed curve. Assume to the contrary that $S^{\prime}$ is a simple closed curve in $X=\bigcup_{i=1}^{n} \stackrel{\circ}{D}_{i}$ and $S^{\prime} \neq S$. As above, we have $\partial_{X}(U) \cup \partial_{X}\left(\stackrel{\circ}{n}_{n}\right) \subset S^{\prime}$. So $S \cap S^{\prime} \neq \emptyset$. Thus there exist arcs $L \subset S$ and $L^{\prime} \subset S^{\prime}$ such that $\stackrel{\circ}{L} \cap S^{\prime}=\emptyset, \stackrel{\circ}{L}^{\prime} \cap S=\emptyset$ and $\operatorname{End}(L)=\operatorname{End}\left(L^{\prime}\right)$. Since $\partial_{X}(U) \cup \partial_{X}\left(\stackrel{D}{n}_{n}\right) \subset S^{\prime} \cap S$, we see that $\stackrel{\circ}{L} \cap\left(\partial_{X}(U) \cup \partial_{X}\left(\stackrel{\circ}{D}_{n}\right)\right)=$ $\stackrel{\circ}{L}^{\prime} \cap\left(\partial_{X}(U) \cup \partial_{X}\left(\stackrel{\circ}{D}_{n}\right)\right)=\emptyset$.

Let $E=\operatorname{End}(L)=\operatorname{End}\left(L^{\prime}\right)$. Since $X=\bigcup_{i=1}^{n} \stackrel{\circ}{D}_{n}=U \cup \stackrel{\circ}{D}_{n}$, there are two cases:

CASE a: $E \subseteq U$. Thus $L \cap \partial_{X}(U)=(\stackrel{\circ}{L} \cup E) \cap \partial_{X}(U)=\emptyset$ and $L \cap U$ $\neq \emptyset$. Since $U$ is a component of $X-\partial_{X}(U)$, we have $L \subset U$. Similarly $L^{\prime} \subset U$. Then $L \cup L^{\prime}$ is a simple closed curve in $U$, which is a contradiction.

CASE b: $E \nsubseteq U$. Then $E \cap \stackrel{\circ}{D}_{n} \neq \emptyset$. Let $x \in E \cap \stackrel{\circ}{D}_{n}$. Since $E$ is the

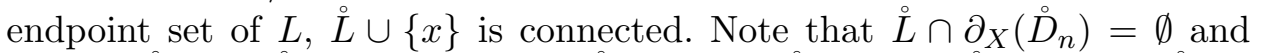

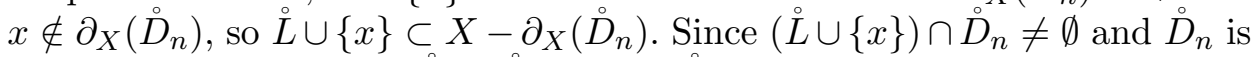
a component of $X-\partial_{X}\left(\stackrel{\circ}{D}_{n}\right), \stackrel{\circ}{L} \cup\{x\} \subset \stackrel{\circ}{D}_{n}$. Similarly, it can also be shown that $\mathscr{L}^{\prime} \cup\{x\} \subset \stackrel{\circ}{D}_{n}$. Thus $L \subset D_{n}$ and $L^{\prime} \subset D_{n}$. Hence $L \cup L^{\prime}$ is a simple closed curve in $D_{n}$, which is a contradiction.

So the assumption is false, i.e., $S^{\prime}=S$.

Let $X$ be a compact metric space with metric $d$. By the hyperspace of $X$, we mean $2^{X}=\{A \mid A$ is a nonempty closed subset of $X\}$ with the Hausdorff metric $d_{\mathrm{H}}$, i.e., $d_{\mathrm{H}}(A, B)=\inf \{\varepsilon>0 \mid B(A, \varepsilon) \supseteq B$ and $B(B, \varepsilon) \supseteq A\}$. It is well known that $\left(2^{X}, d_{\mathrm{H}}\right)$ is a compact metric space. 
Lemma 2.19. Let $X$ be a Peano continuum. Fix an $\varepsilon>0$, and let $\delta=\delta(\varepsilon)$ be as in Lemma 2.8. Suppose $D$ is a strongly free dendrite in $X$ with an arc $A$ being its trunk, $\operatorname{End}(A)=\left\{y_{0}, y_{7}\right\}$ and $\left(y_{1}, \ldots, y_{6}\right)$ is a set of pseudo 7-section points of $A$ from $y_{0}$ to $y_{7}$. Also, suppose that $J$ is an arc in $X, \operatorname{End}(J)=\left\{w_{0}, w_{7}\right\},\left(w_{1}, \ldots, w_{6}\right)$ is a set of pseudo 7 -section points of $J$ from $w_{0}$ to $w_{7}$, and $d\left(w_{0}, w_{7}\right)=d\left(y_{0}, y_{7}\right)=7 \varepsilon$. If $d\left(w_{i}, y_{i}\right)<\delta$ for $i=2,3,4,5$, and $d_{\mathrm{H}}\left(\left[w_{2}, w_{5}\right]_{J},\left[y_{2}, y_{5}\right]_{A}\right)<\delta$, then $\left[w_{3}, w_{4}\right]_{J} \subset\left(y_{2}, y_{5}\right)_{A}$.

Proof. For $i=2,3,4,5$, by Lemma 2.8 there exists an arc $L_{i}$ in $X$ such that $\operatorname{End}\left(L_{i}\right)=\left\{w_{i}, y_{i}\right\}$ and $\operatorname{diam}\left(L_{i}\right)<\varepsilon$. As $d\left(y_{i},\left\{y_{1}, y_{i-1}, y_{i+1}, y_{6}\right\}\right) \geq \varepsilon$, $L_{i} \cap\left\{y_{1}, y_{i-1}, y_{i+1}, y_{6}\right\}=\emptyset$. It follows that $L_{i} \subset D$, and there exists a point $v_{i} \in L_{i} \cap\left(y_{i-1}, y_{i+1}\right)_{A}$ such that $\left[w_{i}, v_{i}\right]_{L_{i}} \cap A=\left\{v_{i}\right\}$.

Since $d_{\mathrm{H}}\left(\left[w_{2}, w_{5}\right]_{J},\left[y_{2}, y_{5}\right]_{A}\right)<\delta$, for each $w \in\left[w_{2}, w_{5}\right]_{J}$ there exist a point $y \in\left[y_{2}, y_{5}\right]_{A}$ and an arc $L$ such that $d(w, y)<\delta$, End $(L)=\{w, y\}$ and $\operatorname{diam}(L)<\varepsilon$. Just as above, we see that $w \in L \subset D$. By the arbitrariness of $w$, we deduce that $\left[w_{2}, w_{5}\right]_{J}$ is an arc in $D$.

But $\left[w_{2}, v_{2}\right]_{L_{2}} \cup\left[v_{2}, v_{5}\right]_{A} \cup\left[v_{5}, w_{5}\right]_{L_{5}}$ is also an arc in $D$ with endpoints $w_{2}$ and $w_{5}$, hence

$$
\left[w_{2}, w_{5}\right]_{J}=\left[w_{2}, v_{2}\right]_{L_{2}} \cup\left[v_{2}, v_{5}\right]_{A} \cup\left[v_{5}, w_{5}\right]_{L_{5}} .
$$

As $d\left(w_{3}, w_{2}\right) \geq \varepsilon$ and $\operatorname{diam}\left(L_{2}\right)<\varepsilon$, we have $w_{3} \notin L_{2}$. Similarly, $w_{4} \notin L_{5}$. Therefore $\left\{w_{3}, w_{4}\right\} \subset\left[v_{2}, v_{5}\right]_{A} \subset\left(y_{1}, y_{6}\right)_{A}$. Noting that $d\left(y_{3},\left\{y_{2}, y_{4}\right\}\right) \geq \varepsilon$, $\operatorname{End}\left(L_{3}\right)=\left\{w_{3}, y_{3}\right\}$ and $\operatorname{diam}\left(L_{3}\right)<\varepsilon$, we have $w_{3} \in\left(y_{2}, y_{4}\right)_{A}$. Similarly, $w_{4} \in\left(y_{3}, y_{5}\right)_{A}$. Hence, $\left[w_{3}, w_{4}\right]_{J} \subset\left(y_{2}, y_{5}\right)_{A}$. This completes the proof.

Lemma 2.20. Suppose $D$ is a strongly free dendrite in $X$ with trunk $A$. If $L$ is an arc in $D$, then there exists a subarc $L^{\prime} \subset L$ such that $D\left(L^{\prime}\right)$ is a strongly free dendrite in $X$.

Proof. Choose any subarc $[\alpha, \beta]_{L} \subset \stackrel{\circ}{L}$. Then $D\left([\alpha, \beta]_{L}\right)$ is the subdendrite of $D$ strung by $[\alpha, \beta]_{L}$. Obviously, $D\left([\alpha, \beta]_{L}\right) \subset D$ is a free dendrite in $X$. Observe that $\{\alpha, \beta\} \subseteq \partial_{X}\left(D\left([\alpha, \beta]_{L}\right)\right) \subseteq \partial_{X}(D) \cup\{\alpha, \beta\}$. Let $\partial_{X}(D)=$ $\operatorname{End}(A)=\{a, b\}$. If $\partial_{X}\left(D\left([\alpha, \beta]_{L}\right)\right) \cap \partial_{X}(D)=\emptyset$, then $\partial_{X}\left(D\left([\alpha, \beta]_{L}\right)\right)=$ $\{\alpha, \beta\}$. Thus if we let $L^{\prime}=[\alpha, \beta]_{L}$, then $D\left(L^{\prime}\right)$ is strongly free in $X$. Otherwise, without loss of generality, suppose $a \in \partial_{X}\left(D\left([\alpha, \beta]_{L}\right)\right)$. Since $[\alpha, \beta]_{L} \subset \stackrel{\circ}{L} \subset \stackrel{\circ}{D}$ and $a \in \partial_{X}(D)$, it follows that $a \in D\left([\alpha, \beta]_{L}\right)-[\alpha, \beta]_{L}$. Suppose $C$ is the component of $D\left([\alpha, \beta]_{L}\right)-[\alpha, \beta]_{L}$ which contains $a$, and $\bar{C} \cap[\alpha, \beta]_{L}=\{x\}$. Then $a \notin D\left([x, \beta]_{L}\right)$. Hence $a \notin \partial_{X}\left(D\left([x, \beta]_{L}\right)\right)$. If $b \notin \partial_{X}\left(D\left([x, \beta]_{L}\right)\right)$, then let $L^{\prime}=[x, \beta]_{L}$; clearly, $\partial_{X}\left(D\left(L^{\prime}\right)\right)=\{x, \beta\}$. So $D\left(L^{\prime}\right)$ is strongly free in $X$. If $b \in \partial_{X}\left(D\left([x, \beta]_{L}\right)\right)$, then similarly we can take a subarc $L^{\prime}=[y, \beta]_{L} \subset[x, \beta]_{L} \subset L$ such that $b \notin D\left(L^{\prime}\right)$. Then $\partial_{X}\left(D\left(L^{\prime}\right)\right)=\{y, \beta\}$. Therefore $D\left(L^{\prime}\right)$ is strongly free in $X$. 
3. Main theorem. To prove the main theorem of this paper, we use the following two lemmas which have been proved in [4]. For convenience of the reader, we prove them again here.

Let $G$ be a group and let $\phi: G \times X \rightarrow X$ be a group action.

Lemma 3.1 ([4]). If $\left\{g_{1}: J \rightarrow J_{1}, g_{2}: J \rightarrow J_{2}\right\}$ is a ping-pong game for $\phi$, then $\left\{g_{1}, g_{2}\right\}$ generates a free subsemigroup of $G$.

Proof. A word $g$ in the semigroup generated by $\left\{g_{1}, g_{2}\right\}$ has the form $g=g_{i_{1}} \cdots g_{i_{l}}$ with $i_{j}=1,2$. Since $g J \subset g_{i_{1}} J \subset J_{i_{1}} \neq J, g$ is not the identity. Hence, $\left\{g_{1}, g_{2}\right\}$ generates a free subsemigroup of $G$.

Lemma $3.2([4])$. If $\left\{g_{1}: J \rightarrow J_{1}, g_{2}: J \rightarrow J_{2}\right\}$ is a ping-pong game for $\phi$, then $h(\phi)>0$.

Proof. Without loss of generality, we can suppose that the symmetric generating set $S=\left\{\sigma_{0}, \ldots, \sigma_{k}\right\}$ for $G$ contains $g_{1}, g_{1}^{-1}, g_{2}, g_{2}^{-1}$. Let $\varepsilon>0$ be the distance between $J_{1}$ and $J_{2}$. Choose any $x \in J$ and set $S_{n}=\left\{g_{i_{1}} \cdots g_{i_{n}} x\right.$ : $\left.i_{j}=1,2\right\}$. The set $S_{n}$ has $2^{n}$ elements. Given any distinct points $y=$ $g_{i_{1}} \cdots g_{i_{n}} x$ and $z=g_{j_{1}} \cdots g_{j_{n}} x$, there exists a least $1 \leq l \leq n$ such that $i_{l} \neq j_{l}$ and $g_{i_{1}} \cdots g_{i_{l-1}}=g_{j_{1}} \cdots g_{j_{l-1}}$. Let $g=g_{i_{1}} \cdots g_{i_{l-1}}$. Then $g^{-1} y=$ $g_{i_{l}} \cdots g_{i_{n}} x \in J_{i_{l}}$ and $g^{-1} z=g_{j_{l}} \cdots g_{j_{n}} \in J_{j_{l}}$. Thus $d\left(g^{-1} y, g^{-1} z\right)>\varepsilon$. It follows that $S(\phi, \varepsilon, N) \geq 2^{N}$ and $h(\phi) \geq \log 2$.

Proof of Theorem 1.1. We can suppose $G$ is countable (see the remark below). Let $c_{0}$ be an expansive constant for $G$-action and $c=c_{0} / 7$. Since $X$ is a Peano continuum containing a free dendrite, by Lemma 2.3 there exists a strongly free dendrite $D$ in $X$. For each $g \in G, g D$ is also a strongly free dendrite and $(g \stackrel{\circ}{D})=g \stackrel{\circ}{D}$ by Lemma 2.6. Let $U=\bigcup_{g \in G} g \stackrel{\circ}{D}$. We argue in two cases.

CASE 1: $U$ contains a simple closed curve $S$. Since $S$ is compact, there is a finite subfamily $\left\{g_{i} \stackrel{\circ}{D}: i=1, \ldots, n\right\}$ of $\{g \stackrel{\circ}{D}: g \in G\}$ covering $S$. By Proposition 2.18, $X=\bigcup_{i=1}^{n} g_{i} \stackrel{\circ}{D}$ and $S$ is the only simple closed curve in $X$. Then $S$ is $G$-invariant, that is, $G S=S$. Since an expansive group action on a simple closed curve must have a ping-pong game (see Propositions 2.10 and 3.1 in [4]), we see that $S$ admits a ping-pong game for the $G$-action, and hence so does $X$.

CASE 2: $U$ contains no simple closed curve. By Proposition 2.16, there is a $G$-invariant open set $W \subseteq U$ such that $\left.G\right|_{W}$ is topologically transitive. By Proposition 2.17, we can find an arc $A^{\prime} \subset W$ all of whose points are transitive points of $\left.G\right|_{W}$. Choose a subarc $A^{\prime \prime} \subset A^{\prime}$ such that $A^{\prime \prime} \subseteq g \stackrel{D}{ }$ for some $g \in G$. Since $g D^{\circ}$ is a strongly free dendrite, from Lemma 2.20, there is a subarc $A \subset A^{\prime \prime}$ such that $A$ is the trunk of a strongly free dendrite. Write $D_{0}=D(A)$. 
Since $U$ is arcwise connected and contains no simple closed curve, for any $x$ and $y$ in $U$, we denote the unique arc in $U$ from $x$ to $y$ by $[x, y]$ and set $(x, y)=[x, y]-\{x, y\}$.

Write $A=[a, b]$. Since $G$ acts on $X$ expansively, there exists $g_{1} \in G$ such that $d\left(g_{1}(a), g_{1}(b)\right)>c_{0}$. Clearly $g_{1}\left(D_{0}\right)$ is a strongly free dendrite with $\left[g_{1}(a), g_{1}(b)\right]$ being its trunk. Write $D_{1}=g_{1}\left(D_{0}\right)$. Select two points $y_{10}, y_{17} \in$ $\left[g_{1}(a), g_{1}(b)\right]$ with $d\left(y_{10}, y_{17}\right)=c_{0}=7 c$. Let $\left(y_{11}, \ldots, y_{16}\right)$ be a set of pseudo 7 -section points of $\left[y_{10}, y_{17}\right]$ from $y_{10}$ to $y_{17}$. Then $d\left(y_{13}, y_{14}\right) \geq c$. Corollary 2.15 yields a subarc $L_{1}=\left[p_{1}, q_{1}\right] \subset\left(y_{13}, y_{14}\right)$ such that $\operatorname{diam}\left(D_{1}\left(L_{1}\right)\right)<1$.

Suppose that for each $i \leq n-1, g_{i}, D_{i}, y_{i 0}, \ldots, y_{i 7}, p_{i}, q_{i}$ and $L_{i}$ have been chosen. For $i=n$, there exists $g_{n} \in G$ such that $d\left(g_{n}\left(p_{n-1}\right), g_{n}\left(q_{n-1}\right)\right)>7 c$. Define $D_{n}=g_{n}\left(D_{n-1}\right)$. Obviously, $\left[g_{n}\left(p_{n-1}\right), g_{n}\left(q_{n-1}\right)\right]$ is a subarc of the trunk of $D_{n}$. Choose $y_{n 0}, y_{n 7} \in\left[g_{n}\left(p_{n-1}\right), g_{n}\left(q_{n-1}\right)\right]$ with $d\left(y_{n 0}, y_{n 7}\right)=7 c$. Let $\left(y_{n 1}, \ldots, y_{n 6}\right)$ be a set of pseudo 7 -section points of $\left[y_{n 0}, y_{n 7}\right]$ from $y_{n 0}$ to $y_{n 7}$. Then $d\left(y_{n 3}, y_{n 4}\right) \geq c$. Take a subarc $L_{n}=\left[p_{n}, q_{n}\right] \subset\left(y_{n 3}, y_{n 4}\right)$ such that $\operatorname{diam}\left(D_{n}\left(L_{n}\right)\right)<1 / n$. By this inductive process, we get a sequence $\left\{g_{n}, D_{n}, y_{n 0}, \ldots, y_{n 7}, p_{n}, q_{n}, L_{n}: n=1,2, \ldots\right\}$ such that, for each $n$ :

(1) $d\left(g_{n}\left(p_{n-1}\right), g_{n}\left(q_{n-1}\right)\right)>7 c$ and $D_{n}=g_{n}\left(D_{n-1}\right)$;

(2) $\left[y_{n 0}, y_{n 7}\right] \subset\left[g_{n}\left(p_{n-1}\right), g_{n}\left(q_{n-1}\right)\right]$ and $d\left(y_{n 0}, y_{n 7}\right)=7 c$;

(3) $\left(y_{n 1}, \ldots, y_{n 6}\right)$ is a set of pseudo 7-section points of $\left[y_{n 0}, y_{n 7}\right]$ from $y_{n 0}$ to $y_{n 7}$ and $d\left(y_{n 3}, y_{n 4}\right) \geq c$

(4) $L_{n}=\left[p_{n}, q_{n}\right] \subset\left(y_{n 3}, y_{n 4}\right)$ with $\operatorname{diam}\left(D_{n}\left(L_{n}\right)\right)<1 / n$.

Let $\delta=\delta(c) \in(0, c / 2]$ be as in Lemma 2.8. Since both $2^{X}$ and $X$ are compact, there are subsequences $\left\{D_{n_{i}}\left(\left[p_{n_{i}}, q_{n_{i}}\right]\right)\right\}_{i=1}^{\infty}$ and $\left\{\left[y_{n_{i} 2}, y_{n_{i} 5}\right]\right\}_{i=1}^{\infty}$ such that $D_{n_{i}}\left(\left[p_{n_{i}}, q_{n_{i}}\right]\right) \rightarrow x_{*}$ as $i \rightarrow \infty$ and for any $i, j \in \mathbb{N}$,

$$
\begin{aligned}
& d_{\mathrm{H}}\left(\left[y_{n_{i} 2}, y_{n_{i} 5}\right],\left[y_{n_{j} 2}, y_{n_{j} 5}\right]\right)<\delta, \\
& \max _{k \in\{2, \ldots, 5\}}\left\{d\left(y_{n_{i} k}, y_{n_{j}}\right)\right\}<\delta .
\end{aligned}
$$

By Lemma 2.19, for each $i, j \in \mathbb{N}$, we have $\left[y_{n_{i} 3}, y_{n_{i} 4}\right] \subset\left[y_{n_{j} 2}, y_{n_{j}} 5\right]$. Now fix a $j \in \mathbb{N}$. For each $i \in \mathbb{N}$, since $\left[p_{n_{i}}, q_{n_{i}}\right] \subset\left(y_{n_{i} 3}, y_{n_{i} 4}\right) \subset\left[y_{n_{j} 2}, y_{n_{j} 5}\right]$ and $\left[p_{n_{i}}, q_{n_{i}}\right] \rightarrow x_{*}$ as $i \rightarrow \infty$, we have $x_{*} \in\left[y_{n_{j} 2}, y_{n_{j}}\right]$. Note that each point in $A$ is a transitive point of $\left.G\right|_{W}$ and $x_{*} \in\left[y_{n_{j} 2}, y_{n_{j}}\right] \subset g_{n_{j}} \cdots g_{1}(A)$, so $x_{*}$ is a transitive point of $\left.G\right|_{W}$.

Since $d\left(y_{n_{j} 3}, y_{n_{j} 4}\right) \geq c$, no matter where $x_{*}$ is in $\left[y_{n_{j} 2}, y_{n_{j} 5}\right]$, we can always choose a subarc $[\alpha, \beta] \subset\left(y_{n_{j} 3}, y_{n_{j} 4}\right)$ such that $\operatorname{diam}([\alpha, \beta])<c / 3$ and $x_{*} \notin[\alpha, \beta]$. By Lemma $2.4, D_{n_{j}}([\alpha, \beta])$ is a strongly free dendrite. Since $x_{*}$ is a transitive point of $\left.G\right|_{W}$, there exists $g_{*} \in G$ such that $g_{*} x_{*} \in$ $D_{n_{j}}([\alpha, \beta])$. By the continuity of $g_{*}$, we can choose a neighborhood $U_{*}$ of $x_{*}$ such that $g_{*}\left(U_{*}\right) \subset D_{n_{j}}([\alpha, \beta])$ and $U_{*} \cap D_{n_{j}}([\alpha, \beta])=\emptyset$. Since $D_{n_{i}}\left(\left[p_{n_{i}}, q_{n_{i}}\right]\right) \rightarrow x_{*}$ as $n_{i} \rightarrow \infty$, there exists some $D_{n_{m}}\left(\left[p_{n_{m}}, q_{n_{m}}\right]\right) \subset U_{*}$. Therefore $g_{*}\left(D_{n_{m}}\left(\left[p_{n_{m}}, q_{n_{m}}\right]\right)\right) \subset D_{n_{j}}([\alpha, \beta])$. On the other hand, let $h=$ 
$\left(g_{n_{m+1}} \cdots g_{n_{m}+1}\right)^{-1}$. Then we have

$$
h\left(D_{n_{m+1}}\left(\left[y_{n_{m+1} 0}, y_{n_{m+1} 7}\right]\right)\right) \subset D_{n_{m}}\left(\left[p_{n_{m}}, q_{n_{m}}\right]\right)
$$

and

$$
g_{*} h\left(D_{n_{m+1}}\left(\left[y_{n_{m+1} 0}, y_{n_{m+1}}\right]\right)\right) \subset D_{n_{j}}([\alpha, \beta]) .
$$

Next we show that both $D_{n_{m}}\left(\left[p_{n_{m}}, q_{n_{m}}\right]\right)$ and $D_{n_{j}}([\alpha, \beta])$ are contained in the closed set $D_{n_{m+1}}\left(\left[y_{n_{m+1} 0}, y_{n_{m+1} 7}\right]\right)$. Since $\left[p_{n_{m}}, q_{n_{m}}\right] \subset\left(y_{n_{m} 3}, y_{n_{m} 4}\right)$ $\subset\left[y_{n_{m+1} 2}, y_{n_{m+1} 5}\right]$, by Lemma 2.5 we have

$$
D_{n_{m}}\left(\left[p_{n_{m}}, q_{n_{m}}\right]\right)=D_{n_{m+1}}\left(\left[p_{n_{m}}, q_{n_{m}}\right]\right) \subset D_{n_{m+1}}\left(\left[y_{n_{m+1} 0}, y_{n_{m+1} 7}\right]\right) .
$$

Similarly, since $[\alpha, \beta] \subset\left(y_{n_{j} 3}, y_{n_{j} 4}\right) \subset\left[y_{n_{m+1} 2}, y_{n_{m+1} 5}\right]$, we have

$$
D_{n_{j}}([\alpha, \beta])=D_{n_{m+1}}([\alpha, \beta]) \subset D_{n_{m+1}}\left(\left[y_{n_{m+1} 0}, y_{n_{m+1} 7}\right]\right) .
$$

Let

$$
J=D_{n_{m+1}}\left(\left[y_{n_{m+1} 0}, y_{n_{m+1} 7}\right]\right), \quad J_{1}=D_{n_{m}}\left(\left[p_{n_{m}}, q_{n_{m}}\right]\right), \quad J_{2}=D_{n_{j}}([\alpha, \beta]) .
$$

Then $\left\{h: J \rightarrow J_{1}, g_{*} h: J \rightarrow J_{2}\right\}$ is a ping-pong game for the $G$-action. Moreover, by Lemma 3.2 the $G$-action has positive geometric entropy.

Since a nilpotent group cannot contain a free subsemigroup, Corollary 1.2 is obvious from Theorem 1.1 and Lemma 3.1.

Remark. If $G$ acts on a compact metric space $X$ expansively, then there exists a countable subgroup $H$ of $G$ such that the induced action of $H$ on $X$ is expansive. In fact, by the expansiveness of the $G$-action, for each $(x, y) \in X \times X, x \neq y$, there is a $g \in G$ such that $d(g x, g y)>c$, where $c$ is the expansive constant for the $G$-action. Thus by the continuity of $g$, there is an open neighborhood $U_{(x, y)}$ of $(x, y)$ in $X \times X$ such that $d\left(g U_{(x, y)}, \Delta_{X}\right)>c$, where $\Delta_{X}$ is the diagonal of $X \times X$. Thus by the Lindelöf property of $X \times X-\Delta_{X}$, there are countable families $\left\{g_{i} \in G: i=1,2, \ldots\right\}$ and $\left\{U_{\left(x_{i}, y_{i}\right)}\right.$ : $i=1,2, \ldots\}$ which covers $X \times X-\Delta_{X}$ such that $d\left(g_{i} U_{\left(x_{i}, y_{i}\right)}, \Delta_{X}\right)>c$. Thus the countable group $H$ generated by $\left\{g_{i}: i=1,2, \ldots\right\}$ is expansive.

Acknowledgments. We are deeply grateful to the referee for his helpful suggestions and detailed revisions of this paper.

Research of E. H. Shi was supported by the National Natural Science Foundation of China (No. 10801103) and by the Natural Sciences Fund for Colleges and Universities of the Jiangsu Province (No. 08KJB110010).

\section{References}

[1] B. F. Bryant, Unstable self-homeomorphisms of a compact space, Vanderbilt Univ. Thesis, 1954.

[2] É. Ghys, R. Langevin and P. Walczak, Entropie géométrique des feuilletages, Acta Math. 160 (1988), 105-142. 
[3] P. de la Harpe, Free groups in linear groups, Enseign. Math. (2) 29 (1983), 129-144.

[4] S. Hurder, Dynamics of expansive group actions on the circle, http://www.math.uic. edu/hurder/.

[5] I. M. Isaacs, Algebra: A Graduate Course, Wadsworth, 1994.

[6] J. F. Jakobsen and W. R. Utz, The nonexistence of expansive homeomorphisms of a closed 2-cell, Pacific J. Math. 10 (1960), 1319-1321.

[7] H. Kato, The nonexistence of expansive homeomorphisms of 1-dimensional compact ANRs, Proc. Amer. Math. Soc. 108 (1990), 267-269.

[8] - , The nonexistence of expansive homeomorphisms of Peano continua in the plane, Topology Appl. 34 (1990), 161-165.

[9] - The nonexistence of expansive homeomorphisms of chainable continua, Fund. Math. 149 (1996), 119-126.

[10] A. Katok, R. de la Llave, Y. Pesin and H. Weiss (eds.), Smooth Ergodic Theory and its Applications, Proc. Sympos. Pure Math. 69, Amer. Math. Soc., Providence, RI, 2001.

[11] J. H. Mai and E. H. Shi, The nonexistence of expansive commutative group actions on Peano continua having free dendrites, Topology Appl. 155 (2007), 33-38.

[12] C. Mouron, Tree-like continua do not admit expansive homeomorphisms, Proc. Amer. Math. Soc. 130 (2002), 3409-3413.

[13] S. B. Nadler Jr., Continuum Theory, Dekker, New York, 1992.

[14] T. O'Brien and W. Reddy, Each compact orientable surface of positive genus admits an expansive homeomorphism, Pacific J. Math. 35 (1970), 737-741.

[15] E. H. Shi, L. Z. Zhou and Y. C. Zhou, The nonexistence of expansive $\mathbb{Z}^{n}$ actions on Peano continua that contain no $\theta$-curves, Topology Appl. 154 (2007), 462-468.

[16] R. F. Williams, A note on unstable homeomorphisms, Proc. Amer. Math. Soc. 6 (1955), 308-309.

Department of Mathematics

Suzhou University

Suzhou 215006, P.R. China

E-mail: ehshi6688@yahoo.com.cn, ehshi@suda.edu.cn

wangsuhuasz@yahoo.com.cn

Received 5 January 2008;

in revised form 12 January 2009 